Justin Rosenberg

\title{
Internationale Beziehungen und die Konsequenzen der Multiplizität
}

\begin{abstract}
Dieser Beitrag greift zwei gegenwärtige, aber bereits länger bestehende Bedenken bezüglich der Disziplin der Internationalen Beziehungen (IB) auf: dass die IB keinen eigenen unverwechselbaren Ansatz zur gesellschaftlichen Analyse entwirft und daher Ideen und Konzepte aus anderen Bereichen importieren muss; und dass die $I B$ aufgrund dieses Defizits zu einer Fragmentierung verurteilt ist, sodass manche bereits das Ende der IB-Theorie vorhersagen. Dieser Beitrag reagiert auf diese Kritiken in drei Schritten. Zunächst wird argumentiert, dass diese problematische Situation letztlich auf die durch den Realismus geprägte negative Definition des Internationalen als politischer Anarchie zurückzuführen ist. Zweitens zeigt der Beitrag, wie eine positive Neudefinition im Sinne einer gesellschaftlichen Multiplizität der IB einen unverwechselbaren Analyseansatz bietet, der voller Konsequenzen für alle Gesellschaftswissenschaften ist. Und schließlich wird vorgeschlagen, dass Multiplizität auch eine neue gemeinsame Basis für die internationale Theorie selbst eröffnet, die sowohl eine optimistischere Neuinterpretation der scheinbaren Fragmentierung der IB als auch eine konstruktive Agenda für die Weiterentwicklung der $I B$ als eigenständige Disziplin bietet.
\end{abstract}

Der Disziplin der Internationalen Beziehungen (IB) werden regelmäßig zwei große Schwächen vorgeworfen. ${ }^{1}$ Erstens hätte sie sich nie als eigenständige Disziplin etabliert, d.h. als Trägerin eines eigenen Ansatzes zur Analyse der sozialen Welt. Bestenfalls eine Interdisziplin, hätte sie nur Ideen von außen importiert; und sicherlich keine große Idee exportiert, die eine internationale Wende in anderen Disziplinen hätte auslösen können (Hoffmann 1959; Halliday 1994; Buzan/Little 2001; Brown 2013). Zweitens, und vielleicht auch aufgrund der Beschleunigung der Importe während der vierten großen Debatte, scheint die IB heute zu fragmentiert, um eine gemeinsame Debatte zuzulassen (Sylvester 2007; Dunne et al. 2013). Ohne gemeinsamen Mittelpunkt steht selbst die Frage im Raum, ob es an der Zeit ist, die Idee der IB an sich zu vergessen (Reiter 2015).

Diese Bedenken sind so alt wie die Disziplin selbst (Kristensen 2016). Und doch wäre die Schlussfolgerung, dass die IB von Natur aus zu einer dauerhaften Unterlegenheit verurteilt ist, falsch. Wie ich im Folgenden darlege, ist das disziplinäre (und transdisziplinäre) Potenzial der IB so groß wie das aller anderen Sozial- oder Geisteswissenschaften - wenn es nur entdeckt und entfesselt werden kann.

1 Dieses Papier ist eine gekürzte und bearbeitete Version von Rosenberg (2016). Der Originalartikel sowie das anschließende Forum sind in den Ausgaben 30: 2 und 31: 1 der Zeitschrift International Relations abgedruckt. 
Mein Argument entfaltet sich in drei Schritten. Zuerst stelle ich die IB in den Kontext anderer, vermeintlich erfolgreicherer Disziplinen, und führe ihr schwaches Standing auf die negative Selbstdefinition über die realistische Idee der politischen Anarchie zurück. Im Gegensatz dazu zeige ich im zweiten Schritt, wie eine positive Definition über gesellschaftliche Multiplizität der IB ihren eigenen Ansatz für gesellschaftliche Analysen bietet, der zudem für andere geistes- und sozialwissenschaftliche Fächer ausgesprochen relevant ist. Schließlich schlage ich vor, dass die Multiplizität auch die Möglichkeit ,einer neuen gemeinsamen Basis für die internationale Theorie“" (Kurki 2017) eröffnet, die sowohl eine optimistische Interpretation der scheinbaren Fragmentierung als auch eine konstruktive Agenda für die Weiterentwicklung der IB als eigenständige Disziplin bietet.

\section{Die IB zwischen den Disziplinen}

Das Studium der menschlichen Welt ist auf eine Reihe von Geistes- und Sozialwissenschaften verteilt. Oftmals beklagen WissenschaftlerInnen diese Fragmentierung und ihre Tendenz zum Provinzialismus, zur Verdinglichung und ideologischen Verzerrung der Realität (Wolf 1982: 7; Mills 1959: 139; Wallerstein 2001: 19). Aber die Arbeitsteilung bringt auch Vorteile. Ob durch Zufall oder Design, jede Disziplin stellt eine bestimmte Dimension der gesellschaftlichen Realität in den Vordergrund und macht sie zum Gegenstand eines systematischen Zugangs. Durch die Formulierung neuer Konzepte, Methoden und Theorien zur Erforschung dieser Dimension ermöglicht die fachliche Spezialisierung eine viel tiefergehende Analyse.

Die Geografie, zum Beispiel, ist auf die räumliche Dimension der menschlichen und natürlichen Existenz spezialisiert - sowohl was Raum für die Gesellschaft bedeutet als auch wie Raum selbst zu verschiedenen Zeiten und an verschiedenen Orten auf unterschiedliche Weise sozial konstruiert wird. Ihre Grundprämisse ist die Tatsache, dass sowohl die menschliche als auch die natürliche Welt im dreidimensionalen Raum existieren und dass dies für die physische und soziale Realität von Bedeutung ist.

Ebenso ordnet sich die Geschichtswissenschaft letztlich unsere Existenz in der Zeit unter - dies erklärt ihre Beschäftigung mit historischer Spezifität, Chronologie, kausaler Sequenzierung und narrativen Formen der Erklärung. ,Zeit [...] ist der besondere Gegenstandsbereich des Historikers/der Historikerin. [...] Sie ist der Boden, in dem wir graben, das Element, aus dem die Geschichte selbst entspringt“ (Guldi/Armitage 2014: 15). Zeitlichkeit ist für die Geschichtswissenschaft, was Räumlichkeit für die Geografie ist.

Ähnlich gründet sich die Soziologie darauf, dass das individuelle menschliche Leben immer in breiteren Strukturen sozialer Beziehungen stattfindet, die sowohl aggregierte systemische Effekte als auch lokale molekulare Definitionen menschlicher Akteure in spezifischer Weise hervorrufen. Es ist kein Zufall, dass die AkteurStruktur-Debatte in dieser Disziplin ständig wiederkehrt. Denn ,,die Idee der Sozi- 
alstruktur steht im Mittelpunkt der Soziologie als wissenschaftlichem Unternehmen“ (Smelser 1988: 103).

Um ein letztes Beispiel zu nennen: Vergleichende Literaturwissenschaft ist nicht nur das Studium der verschiedenen nationalen Traditionen des kreativen Schreibens. Auf einer tieferen Ebene geht es auch um die Eigenschaften der Sprache selbst: Wie ihr metaphorisches und metonymisches Potenzial mobilisiert wird, um menschliche Bedeutungen zu schaffen; und wie im weiteren Sinne die soziale Welt insgesamt, die auch sprachlich vermittelt und produziert wird, Eigenschaften der Textualität aufweist, die zur hermeneutischen Dekonstruktion und Analyse einladen.

In jedem dieser vier Fälle beruht eine akademische Disziplin auf einem spezifischen Merkmal der sozialen Realität - Räumlichkeit, Zeitlichkeit, soziale Struktur und Textualität. Diese Spezialisierung kann sicherlich eine Formel für Fragmentierung und Verdinglichung sein. Und doch ist sie auch entscheidend für das Geheimnis ihres transdisziplinären Potenzials. Gerade weil jede von ihnen etwas in Besitz genommen hat, das der sozialen Welt in der Tat gemein ist, können die Konzepte einer jeden Disziplin unvermittelt reisen und quer durch die Geistes- und Sozialwissenschaften angewendet werden.

Wir sehen, wie sich dies ständig wiederholt. In den 1980er Jahren entwickelte sich in der Geografie ein spatial turn, der in einer Disziplin nach der anderen aufgegriffen wurde (Gregory/Urry 1985). Einige Jahrzehnte zuvor entstand durch die Zerlegung der historischen Zeit durch die Annales-Schule ein Konzept der historischen Konjunkturen, das weit über die Disziplin der Geschichtswissenschaft hinaus verwendet wurde. Immanuel Wallersteins interdisziplinäres Projekt der Weltsystemanalyse begann als Antwort auf die spezielle Frage der Soziologie: Was ist ein soziales System? In jüngster Zeit haben LiteraturtheoretikerInnen eine Schlüsselrolle bei der Entstehung und Verbreitung der postkolonialen Theorie gespielt. In den postkolonialen Studien hat sich die Vergleichende Literaturwissenschaft durch ihren besonderen Fokus auf die Sprache zu einer führenden Stimme im interdisziplinären Gespräch über die Textualität der sozialen Welt entwickelt. Ein ähnlicher Punkt könnte zu der ethnografischen Methode angeführt werden, die im Rahmen der spezialisierten Kulturwissenschaft der Anthropologie heute in den Sozialwissenschaften eine viel größere Bedeutung hat.

Es stellt sich also die Frage: Welches allgemeine Merkmal ist die Spezialität der IB, die es uns ermöglicht, mit anderen Disziplinen in unserer eigenen Sprache über ihren jeweiligen Gegenstandsbereich zu sprechen?

Hier wird die gegenwärtige Besonderheit der IB als Disziplin am deutlichsten. Diejenigen IB-WissenschaftlerInnen, die die Besonderheit des Internationalen geltend gemacht haben, taten dies weitgehend durch Negation: Internationale Beziehungen unterscheiden sich von anderen Aspekten des gesellschaftlichen Lebens durch das Fehlen einer zentralen Autorität. Auf diese Weise ist das Internationale mit einem engen Verständnis des politischen Realismus verbunden. Aber warum sollte dies die IB daran hindern, Ideen zu entwickeln, die in andere Disziplinen übertragen werden können? 
Betrachten wir das Beispiel von Kenneth Waltz. Er hat durchaus die Besonderheit des Internationalen herausgearbeitet. Aber sein Werk hat zwei Eigenschaften, die es daran hindern, eine transdisziplinäre Bedeutung zu erlangen. Erstens definierte er die internationale Theorie als internationale politische Theorie: Sie umfasste ausdrücklich keine breitere Bedingung der Internationalität mit Auswirkungen über die Politikwissenschaft hinaus. Stellen wir uns eine Disziplin der Geografie oder Literaturwissenschaft vor, die auf diese Weise gefangen und daher nicht in der Lage wäre, die breitere Bedeutung von Räumlichkeit oder Textualität für die soziale Realität deutlich zu machen.

Zweitens konzeptualisierte Waltz das Internationale als vom innerstaatlichen Bereich getrennt und diesem entgegengesetzt. Über diese innerstaatliche Welt hatte der Neorealismus - im wahrsten Sinne des Wortes - nichts zu sagen, außer zu bemerken, wie sehr sie sich von der Welt der internationalen Politik unterscheidet. So ist einerseits ,alle theoretische Entwicklung [in der IB] seit 1979 eine Reihe von Fußnoten zu Waltz“ (Dunne et al. 2013: 413); andererseits wird „Waltz' Werk [...] bestenfalls vage in der Politikwissenschaft im Allgemeinen wahrgenommen und ist im breiteren Feld der Geisteswissenschaften fast gänzlich unbekannt" (Brown 2013: 484).

Natürlich ist der Realismus nicht der einzige Ansatz in der IB. Zahlreiche andere haben sowohl die enge, staatszentrierte Definition des Internationalen als auch die Idee einer Autonomie der Geopolitik abgelehnt. Die schiere Bandbreite dieser Alternativen - von Liberalismus, Marxismus und Feminismus bis hin zu Konstruktivismus, Postkolonialismus, Queer-Theorie usw. - macht einen Großteil der Lebendigkeit der heutigen IB aus. Aber wie viele dieser Herausforderer haben den Realismus durch eigene Theorien ersetzt, die auf den einzigartigen Eigenschaften des Internationalen basieren? Die Antwort, so scheint es, lautet: keine. Vielleicht liegt das daran, dass sie die Idee des Internationalen als Teil des giftigen Erbes des Realismus betrachten (mit seinem Beharren auf der anarchischen Unvermeidlichkeit von Machtpolitik und Krieg). Das ist verständlich, kommt aber zu einem hohen Preis. Denn es führt zu dem Schluss, dass die internationalen Angelegenheiten von anderen Aspekten der sozialen Welt geprägt sein müssen und daher am besten durch Ideen interpretiert werden können, die aus den Disziplinen stammen, die diese Aspekte untersuchen. Leider wird dadurch nur der subalterne Status der IB als Disziplin verstärkt: Wenn wir erklären, dass die Soziologie oder Geschichtswissenschaft oder Anthropologie den Schlüssel zum Verständnis des Gegenstandes der IB hält, dann machen wir die IB letztendlich zu einem Teilbereich der Soziologie, Geschichtswissenschaft oder Anthropologie. Der Nachteil der wunderbaren Offenheit der IB gegenüber anderen Disziplinen besteht darin, dass wir ohne eigene tiefe Ontologie praktisch zum Teilbereich aller werden.

Insofern ist es nicht verwunderlich, dass keine großen Ideen aus der IB nach außen gelangt sind. Bei solchen Ideen müsste es um die einzigartige und allgemeine Bedeutung des Internationalen für die menschliche Welt gehen. Aber die RealistInnen haben das Internationale für diese Rolle zu eng definiert. Und die AntirealistInnen haben sich von der Einzigartigkeit der Internationalität ferngehalten, weil sie 
sie mit realistischen Behauptungen über Anarchie und Machtpolitik verbinden, die $\mathrm{zu}$ widerlegen sie entschlossen sind. Was auch immer die Bedeutung des Internationalen für die anderen Geistes- und Sozialwissenschaften sein mag, sie ist weitgehend unsichtbar geblieben, auch für die IB selbst. Lehrbücher der Politik, Soziologie und Ökonomie beginnen regelmäßig mit der allgemeinen Bedeutung ihres Fachgebiets: Es gibt, so behaupten sie, eine Politik, eine Soziologie, eine Ökonomie von allem. IB-Lehrbücher hingegen erheben selten solche Ansprüche.

Aber liegt das daran, dass die IB wirklich keine echte Disziplin wie die anderen ist? Um das herauszufinden, müssen wir uns noch einmal die grundlegende Frage stellen: Wenn die Geografie unsere Existenz im Raum untersucht und die Soziologie die Beziehungsqualität des menschlichen Lebens analysiert, gibt es ein allgemeines Merkmal der sozialen Welt, das der IB eine gleichwertige ontologische Prämisse bietet?

Wie sehr wir es auch drehen und wenden, das Wort international setzt immer denselben grundlegenden Umstand voraus: nämlich, dass die menschliche Existenz nicht einheitlich, sondern vielfältig ist; sie ist über zahlreiche interagierende Gesellschaften verteilt. Diese Vielfalt ist die grundlegende Tatsache der menschlichen Welt, die die Existenz der IB als akademische Disziplin rechtfertigt. Keine andere Disziplin stellt diese Tatsache in den Mittelpunkt - nicht einmal die Politikwissenschaft. Es gibt, sagt Adrian Leftwich, ,,ein vorrangiges Anliegen derjenigen, die Politik studieren, und das ist ein Interesse an Macht, politischer Macht - und ihren Auswirkungen“ (2004: 19). Macht wiederum findet sich überall dort, „wo zwei oder mehr Menschen an einer kollektiven Aktivität beteiligt sind“" (2004: 100). Die Politik bezieht sich damit auf Macht als universelles Merkmal der menschlichen Gesellschaft. Diese Gesellschaft beinhaltet internationale Beziehungen, aber im Gegensatz zu der IB kann Politik auch in einer Welt mit einer einzigen Gesellschaft existieren. Die gesellschaftliche Multiplizität ist daher für die IB in einer Weise entscheidend, wie sie es nicht für die Politikwissenschaft ist.

Natürlich müssen sich alle Sozial- und Geisteswissenschaften mit den Konsequenzen dieser Tatsache auseinandersetzen, ebenso wie die IB unweigerlich auf die Bedeutung von Räumlichkeit, Textualität und so weiter in ihrem eigenen Gegenstandsbereich stößt. In den letzten Jahren haben KulturanthropologInnen beispielsweise die Bedeutung der primitiven Kriegsführung und anderer Interaktionen in Prozessen der frühen Staatsbildung betont - ein Schwerpunkt, der zwangsläufig Multiplizität voraussetzt. Dies ändert jedoch nichts daran, dass die Anthropologie sich durch ihre Analyse der menschlichen Welten durch das Prisma der Kultur auszeichnet. Wie Eric Wolf einmal argumentierte, wäre eine Anthropologie ohne Interaktionsvielfalt sicherlich eine schlechte Anthropologie (Wolf 1982: 15, 18). Anthropologie ohne Kultur wäre jedoch überhaupt keine Anthropologie mehr. Denn Kultur, wie Clifford Geertz es ausdrückte, ist das Konzept, „um welches die Disziplin der Anthropologie an sich entstanden ist" (1993: 4). In ähnlicher Weise entsteht die Disziplin der IB aus der Tatsache der gesellschaftlichen Multiplizität im menschlichen Leben. Das ist unsere einzigartige ontologische Prämisse. 
Und sie hat bemerkenswerte Folgen für alle Sozialwissenschaften. Einige dieser Folgen sind uns bereits ansatzweise bekannt, allerdings nur unter einem negativen Vorzeichen: dem Zeichen des Fehlens einer übergreifenden Regierung. Wir kennen sie noch nicht unter dem positiven Zeichen der Internationalität - der Ko-Präsenz multipler, interagierender Gesellschaften. Und wenn die IB ihre eigene Stimme unter den Disziplinen finden soll, dann ist der Zeichenwechsel entscheidend. Welche Konsequenzen folgen daraus?

\section{Die Konsequenzen der Multiplizität}

\subsection{Koexistenz}

Die erste und tiefgehendste Konsequenz ist zugleich auch die einfachste: Auf ihrer höchsten Ebene gipfelt die menschliche Welt nicht in einer einzigen Autorität; aber sie geht auch nicht einfach in einen leeren Raum über, sondern öffnet sich in ein Feld koexistierender Gesellschaften.

Dieses Feld der Koexistenz fügt der sozialen Realität jenseits der internen Strukturen jeder einzelnen Gesellschaft eine völlig neue Dimension hinzu - die die menschliche Welt nicht nur vergrößert, sondern auch eine völlig neue $A r t$ sozialer Phänomene begründet. Gesellschaften koexistieren in einem ,dezentralisierten [...] Milieu“ (Hoffmann 1959: 347), dessen Ordnung letztlich nicht auf der Konzentration politischer Autorität und legitimer Gewalt, sondern auf ihrer radikalen Verteilung auf mehrere Zentren beruht. Damit entsteht ein Umfeld, in dem sich, unbegrenzt von einer höheren Autorität, die dynamische Logik der Multiplizität als soziale Bedingung in einem einzigartigen Maße durchsetzt. Hier entstehen Formen des politischen Handelns - Diplomatie und Kriegsführung -, die für die Ordnung der menschlichen Welt grundlegend sind und doch innerhalb von Gesellschaften keine exakten Entsprechungen haben.

Diese politische Konsequenz der Multiplizität ist von immenser Bedeutung und dominiert verständlicherweise orthodoxe realistische Darstellungen des Internationalen. Aber es ist nicht nur die Politik, die besondere Merkmale annimmt, wenn sie über mehrere Gesellschaften hinweg operiert. „Solange es Länder gibt“, schreibt Thomas Pugel, „wird die internationale Ökonomie ein Analysekomplex sein, der sich vom Rest der Ökonomie unterscheidet" (2015: 1). Auch die Prämissen der ethischen Argumentation ändern sich angesichts moralischer Verpflichtungen, die sich über mehrere Gesellschaften erstrecken (Walzer 1994: 1). Selbst Realitätswahrnehmungen operieren in einem Kontext, in dem „es noch keiner Macht gelungen ist, autoritative Bedeutungen durchzusetzen“ (Seth 2011: 182). Und wie die wachsende Literatur über ungleichzeitige und kombinierte Entwicklung zeigt, fügt die Koexistenz den Prozessen der historischen und kulturellen Entwicklung und Veränderung auch eine völlig neue Dimension der sozialen Kausalität hinzu (Rosenberg 2013). 
So schafft Multiplizität das Internationale als eine vielseitige Dimension der sozialen Welt. Und es ist die zentrale Aufgabe der IB, diese Dimension in den Mittelpunkt zu stellen und als Studienobjekt zu konstruieren. Wird damit das transdisziplinäre Potenzial der IB freigesetzt? Um das herauszufinden, müssen wir die weiteren Folgen der Multiplizität systematisch untersuchen.

\subsection{Differenz}

Die zweite Folge ist die Differenz: Denn die quantitative Multiplizität der Gesellschaften ist auch eine qualitative. Wir sehen dies empirisch: Gesellschaften unterscheiden sich auf vielfältige Weise - in Größe, Macht, Kultur, Geschichte und so weiter. Aber Differenz ist auch eine notwendige Folge der Multiplizität selbst. Warum?

Einerseits unterscheiden sich verschiedene Gesellschaften notwendigerweise in ihrer geografischen Lage und unterliegen damit sowohl unterschiedlichen Einflüssen physischer (Klima, Erde) Art wie auch sozialen und politischen Einflüssen, die aus ihrer spezifischen Position in Beziehung zu anderen Gesellschaften entspringen. Andererseits entsteht Differenz aber auch dadurch, dass die Verteilung der sozialen Entwicklung über mehr als eine Gesellschaft es ihr ermöglicht, verschiedene Formen an verschiedenen Orten gleichzeitig anzunehmen. Eines der charakteristischsten Merkmale des Menschen als Spezies ist die Fähigkeit, unsere soziale Existenz auf radikal unterschiedliche Weise zu konstruieren und diese Wege selbst der historischen Entwicklung und Veränderung zu unterziehen. SoziologInnen argumentieren seit Langem, dass ,diese Tatsache der Diversität [...] das bedeutendste Merkmal der menschlichen sozialen Organisation darstellt“ (Megarry 1995: xii). Aber die Multiplizität transformiert diese Eigenschaft: Sie ist nicht nur eine vergleichende Tatsache über verschiedene Gesellschaften zu unterschiedlichen Zeiten und an verschiedenen Orten, sondern findet nun auch Ausdruck in einer konkreten Konfiguration verschiedener Gesellschaften, die in Raum und Zeit koexistieren. Auf diese Weise verankert das Internationale Differenz und Multilinearität in der Natur der globalen sozialen Entwicklung.

\subsection{Interaktion}

Bei der Multiplizität geht es jedoch nicht nur um Koexistenz und Differenz. Sie zwingt Gesellschaften auch zur Interaktion. Denn sie setzt ihnen allen eine gemeinsame Bedingung: Jede Gesellschaft sieht sich mit der Tatsache konfrontiert, dass die menschliche Welt über sie selbst hinausgeht - und darin liegen sowohl Gefahren als auch Chancen.

Gefahren, weil externe Prozesse die Interessen oder gar das Überleben einer Gesellschaft bedrohen können. Im Laufe des 19. Jahrhunderts wurden zahlreiche asiatische und afrikanische Gesellschaften überwältigt, weil die industrielle Revolution 
die Macht anderer - ihnen vorher kaum bekannter - (europäischer) Staaten vergröBert hatte.

Aber Multiplizität bietet auch Chancen: Koexistenz und Differenz bedeuten, dass die Entwicklungsmöglichkeiten einer jeden Gesellschaft nie ausschließlich durch ihre innere soziale Struktur und ihren kulturellen Horizont definiert sind. Die Existenz anderer Gesellschaften schafft die Grundlage sowohl für den Reichtum durch Handel als auch für den Import von Wissen und Ressourcen, die durch unterschiedliche Entwicklungsmuster an anderer Stelle entstehen. Ende des 20. Jahrhunderts etwa hat China seine Position in der Welt dramatisch verändert, indem es Wirtschaftsmodelle, Produktionstechnologien, Investmentfonds und Märkte nutzte, die überwiegend in anderen Gesellschaften entwickelt worden waren (Rosenberg/ Boyle 2019). Tatsächlich kann, wie Karl Marx einst bemerkte, allein schon das Wissen um die Existenz anderer Gesellschaften, in denen das soziale Leben auf andere Weise geordnet ist, zu einer Quelle des inneren Wandels werden (Marx/Engels 1970: 52).

Multiplizität führt also zu Interaktion, weil Gesellschaften ihre äußere Umwelt mit diplomatischen und militärischen Mitteln verwalten müssen, um zu überleben; und wenn sie von den Möglichkeiten der Differenz profitieren wollen, müssen sie auch materiell und kognitiv mit anderen interagieren. Alle modernen Gesellschaften sind permanent mit beidem beschäftigt. Das sind die internationalen Beziehungen, die der Mainstream-IB-Theorie bekannt sind - Geopolitik und Interdependenz. Aber damit sind die Auswirkungen der Multiplizität noch nicht erschöpft.

\subsection{Kombination}

Und das liegt daran, dass die Interaktion eine vierte Konsequenz mit sich bringt: Keine Gesellschaft durchläuft eine lineare und in sich geschlossene Geschichte. Alle Gesellschaften stellen daher immerfort eine Kombination lokaler Entwicklungsmuster mit äußeren Einflüssen und Belastungen aller Art dar.

Wir haben bereits zwei dramatische Beispiele dafür erwähnt: Durch den Prozess des europäischen Imperialismus entstand die Mehrheit der modernen Staaten als interaktive Verschmelzung indigener sozialer Formen mit der westlichen kolonialen/ nationalen Staatsstruktur; und Chinas gesamte moderne Geschichte - von der heutigen „importierten industriellen Revolution“ (Vogel 2011: 464), zurück durch drei Jahrzehnte des marxistischen Leninismus mit chinesischen Merkmalen bis hin zum Jahrhundert der Erniedrigungen, das China von 1839 an aufgezwungen wurde hat sich im Zuge all dieser Wechselwirkungen zu der spezifischen Mischung imperialer, leninistischer und kapitalistischer Ausprägung entwickelt, die heutige BeobachterInnen so sehr verwirrt. Andere Beispiele bieten sich an: In Saudi-Arabien wurden externe Prozesse der wirtschaftlichen und technologischen Entwicklung paradoxerweise auf eine stammespolitische Struktur aufgepfropft, während der moderne Iran eindeutig westliche und einheimische Elemente in der Natur der Islamischen Republik (Matin 2013) verschmolzen hat. Zudem ist dieser Kombinations- 
prozess nicht sonderlich modern. Die Ursprünge des russischen Staates im 10. Jahrhundert liegen beispielsweise in der Kombination von wikingischen Handelsaktivitäten mit dem Erbe des orthodoxen Christentums, des kyrillischen Alphabets und des byzantinischen Handelsrechts, die alle durch die merkantile und geopolitische Interaktion mit Konstantinopel absorbiert wurden (Bozeman 1994: 344-6). Was könnte zudem englischer erscheinen als die englische Sprache? Und doch wissen wir, dass es sich tatsächlich um eine Mischung der lateinischen, angelsächsischen, nordischen und französischen Sprachen handelt, die von Englands Eroberern mitgebracht wurden - ganz zu schweigen von den weiteren Entwicklungen, die sie danach durch ihre kolonialen und postkolonialen Aktivitäten und Verschmelzungen erfahren hat (Hogg/Denison 2006). Schließlich verfolgen WeltgeschichtswissenschaftlerInnen die Rolle der interaktiven Kombination bis zum Beginn der aufgezeichneten Geschichte selbst zurück: Die Prozesse der sozialen Schichtung und Staatsbildung, die zur Entstehung des antiken Sumers führten, fanden an der mesopotamischen Kreuzung paläolithischer Handelsrouten statt; und in einer Überschwemmungsebene gelegen, war Sumer von Anfang an auf den Handel mit Metallen, Hölzern und Edelsteinen, die eine wesentliche Rolle für die Entwicklung dieser Zivilisation spielten, angewiesen (McNeill/McNeill 2003: 45-51).

Nicht umsonst schrieb Friedrich Tenbruck, dass ,die innere Gliederung von Gesellschaften überall durch externe Faktoren bedingt, bestimmt oder sogar entstanden ist, so daß jede Gesellschaft auf andere bezogen, mit ihnen verspannt oder sogar von Vergesellschaftungen durchzogen ist, die zu allen querstehen“ (1989: 428).

Das hat dramatische Folgen für die IB wie auch für alle anderen Sozialwissenschaften. Es zeigt, dass die internationale Dimension nicht nur eine Frage der Außenbeziehungen ist: Durch die Interaktion greift die Multiplizität in die innere Verfasstheit der Gesellschaften selbst ein. Und so hält die IB, wenn wir uns dessen nur bewusst werden, die Lösung für das Problem des methodischen Nationalismus bereit - der (meist unbewussten) Annahme, dass einzelne Gesellschaften oder Gesellschaftsformen allein unter Bezugnahme auf ihre inneren Merkmale verstanden werden können. Dies ist ein Problem, das bis zu den ursprünglichen Schriften der klassischen Sozialtheorie zurückgeht und die Geistes- und Sozialwissenschaften auch heute noch belastet (Nisbet 1969; Skocpol 1973; Bauman 1992; Beck 2007).

\subsection{Dialektischer Wandel}

Wenn menschliche Gesellschaften multipel, vielfältig und interaktiv sind, dann folgt daraus schließlich auch, dass der Prozess der Weltentwicklung nicht unilinear oder multilinear sein kann. Es muss sich um einen gänzlich dialektischen Prozess handeln - der durch Interaktionen zwischen verschiedenen Gesellschaften geprägt ist und nicht nur der Logik ihrer eigenen internen Entwicklung folgt.

Betrachten wir dieses Beispiel: 1620 schrieb Francis Bacon, dass die Auswirkungen dreier wichtiger Erfindungen - Schießpulver, Druckmaschine und Magnetkompass - die moderne Welt von der Vergangenheit trennten (Bacon 1960: 118). Ge- 
meinsam, so Bacon, hätten diese Erfindungen mehr als jede Macht oder Religion dazu beigetragen, Europa aus dem finsteren Mittelalter zu befreien. Bacon war unbekannt, dass alle drei ihren Ursprung in China hatten und durch Prozesse des indirekten Handels und der Kommunikation nach Europa gelangt waren (Hobson 2004: 123, 185-186). Aber dies ist nicht nur ein Fall von allgemeiner Vernetzung: Diese chinesischen Erfindungen gelangten in Europa in ein anderes soziales Umfeld, in dem sie sich daher in völlig neue Richtungen entwickelten, mit Ergebnissen, die sie in China nie hatten. Dasselbe gilt für den Transfer des klassischen griechischen Wissens aus der arabischen Welt nach Europa zu Beginn der Renaissance - oder auch für die ursprüngliche Übersetzung der griechischen Philosophie ins Arabische dreihundert Jahre zuvor (Gutas 1998). In all diesen Fällen hatte der dialogische Transfer von einer Gesellschaft zur anderen einen neuen Entwicklungsprozess zufolge, der den weiteren Verlauf der Weltentwicklung selbst beeinflusste. Aus dieser Perspektive erweist sich selbst der Aufstieg des Westens als Ergebnis einer dialektischen Kausalität, die durch die Wechselwirkungen mehrerer sozialer Formationen hervorgerufen wurde.

Nicht nur methodologischer Nationalismus, sondern auch Eurozentrismus, Unilinearität und Teleologie in der historischen Erklärung können daher durch die Ontologie der IB - die Ontologie der gesellschaftlichen Vielfalt und ihrer Folgen - überwunden werden.

Und was für die Weltgeschichte im Allgemeinen gilt, gilt auch für ihre spezialisierten kulturellen Prozesse. Im 19. Jahrhundert leisteten brasilianische SchriftstellerInnen einen einzigartigen Beitrag zur Weiterentwicklung des Romans als literarisches Genre. Doch wie Roberto Schwarz gezeigt hat, war dieser Beitrag untrennbar mit dem zwischengesellschaftlichen Prozess verbunden, durch den diese ursprünglich europäische Literaturgattung in ein radikal anderes soziales und kulturelles Umfeld überführt worden war, wodurch eine neue Konfiguration von literarischer Form und sozialstrukturellem Kontext entstand (Schwarz 1992; vgl. u.a. Rosenberg 2016: 147-8). Ähnliche Argumente könnten sicherlich über die Bedeutung der erzwungenen Migration der AfrikanerInnen nach Amerika für die moderne Musik oder die Rolle des Imperialismus für die Entwicklung moderner kulinarischer und anderer Geschmäcker angeführt werden.

Koexistenz, Differenz, Interaktion, Kombination, Dialektik: Was sagen uns diese fünf Punkte über den Gegenstandsbereich der IB? Sie zeigen, dass die Folgen gesellschaftlicher Multiplizität einen spezialisierten IB-Fokus auf die menschliche Welt begründen, der für die Sozial- und Geisteswissenschaften von allgemeiner Bedeutung ist. $\mathrm{Zu}$ ihren Aufgaben gehört auf jeden Fall der Bereich der Geopolitik und Interdependenz, den realistische und liberale Theorien behandeln. Sie umfasst aber auch die Auswirkungen der gesellschaftlichen Vielfalt auf alle sogenannten inneren Aspekte des gesellschaftlichen Lebens: auf soziale Strukturen, Wirtschaftssysteme, intellektuelle Produktion, kulturelle Phänomene und so weiter. Um es anders auszudrücken: Eine Disziplin der IB sollte sicherlich versuchen zu verstehen, was in der internationalen Politik passiert; sie sollte aber auch die Bedeutung der 
gesellschaftlichen Multiplizität für die gesamte soziale Welt herausarbeiten - so wie die Geografie für den Raum, die Anthropologie für die Kultur und so weiter.

So gesehen muss die IB nicht mehr die stille Außenseiterin im Gespräch der Geistes- und Sozialwissenschaften bleiben. Anstatt nur über die Bedeutung von Klasse, Geschlecht, Sprache und so weiter für die IB zu sprechen, können wir auch die Bedeutung des Internationalen für Klasse, Geschlecht und Sprache untersuchen.

\section{Das unvollendete Puzzle der Internationalen Beziehungen}

Die Frage bleibt jedoch: Kann diese breitere Konzeption der IB - IB als die vielfältigen Folgen der Multiplizität - eine neue gemeinsame Basis innerhalb der IBTheorie für ein scheinbar weitgehend fragmentiertes Feld bieten?

Darauf gibt es eine zweiteilige Antwort. Erstens müssen wir erkennen, inwieweit Multiplizität bereits, wenn auch stillschweigend, als gemeinsames Objekt der IBTheorie fungiert. Und zweitens müssen wir überlegen, welche gemeinsame Agenda für die künftige intellektuelle Entwicklung sich aus der Analyse dieser Idee ergibt.

Wir können den ersten Teil angehen, indem wir Christine Sylvesters Metapher vom „Lager der IB“ (2007: 551) betrachten. Vor den 1980er Jahren, sagt sie, sei die IB-Theorie zwar ausgesprochen begrenzt und eng gewesen, aber ihre drei oder vier unterschiedlichen Ansätze hätten zumindest am selben Lagerfeuer gesessen: Sie hatten einen gemeinsamen Gegenstandsbereich und haben direkt miteinander diskutiert. Heutzutage ist die IB dagegen sehr viel größer und breiter, aber auch fragmentierter. Konstruktivismus, Poststrukturalismus, Feminismus, Postkolonialismus und so weiter haben alle ihre eigenen Lagerfeuer entzündet, an denen sie nur noch untereinander und über völlig verschiedene Gegenstände diskutieren. Es gibt also kein gemeinsames Unterfangen der internationalen Theorie mehr und Sylvester akzeptiert daher die weit verbreitete Ansicht des Endes der IB-Theorie in einem einheitlichen Sinne (2007: 562).

Und sie hat ihre eigene kreative Antwort darauf. Obwohl wir die Idee der Wiederherstellung eines gemeinsamen Diskurses aufgeben müssen, können wir doch dem Beispiel von Collagen in der Kunst folgen. In einer Collage, sagt sie, werden unverbundene Objekte oder Fragmente aus dem Alltag auf einer fremden Oberfläche nebeneinandergelegt und stellen dadurch unerwartete Verbindungen und Beziehungen her, die etwas Neues offenbaren. Das Gleiche könnten wir mit den theoretischen Fragmenten der IB tun: zwei oder mehr Theorien nebeneinanderlegen und sehen, was diese Nähe Unerwartetes offenbart (Sylvester 2007: 571-573).

Das ist eine überzeugende Metapher. Aber folgen wir dem Gedankenexperiment einen Schritt weiter. Was wäre, wenn wir mit dem Bau unserer Collage begännen und feststellten, dass die Fragmente nicht ganz so zufällig geformt sind, wie ursprünglich angenommen? Was wäre, wenn sich herausstellen sollte, dass es sich tatsächlich um Puzzlestücke handelt - nicht um das ganze Set, aber genug für ein gro- 
bes Gesamtbild, bei dem nur einige Stücke fehlen und der Rest richtig zusammengesetzt werden müsste?

Vermutlich ist dies der Punkt, an dem die IB-Theorie heute steht. Schließlich definiert der Realismus das Internationale als Anarchie, was andersherum aber auch als spezifisch politische Folge der Multiplizität verstanden werden kann. Richtig herum könnte dieses Stück zu anderen Puzzleteilen passen, die ursprünglich völlig inkompatibel aussahen. Der Poststrukturalismus scheint zunächst aus einem anderen Puzzle zu stammen. Aber eigentlich geht es im Frühwerk von Richard Ashley, David Campbell und R.B.J. Walker immer um eine spezifische Lesart der Multiplizität und ihre Bedeutung für die Politik (Ashley 1984; Walker 1992; Campbell 1992). Auch der IB-Konstruktivismus begann als Ablehnung der geschlossenen realistischen Konzeption von politischer Multiplizität aufgrund einer Analyse interaktiver Identitätsbildung (Wendt 1992). Für den Marxismus in der IB bestand die zentrale Herausforderung unterdessen immer in der Frage, wie der durch das Kapital geschaffene einheitliche Weltmarkt mit der dauerhaften politischen Vielfalt des Staatensystems in Einklang gebracht werden kann (Callinicos 2007). Auch der Feminismus hat nicht nur die geschlechtsspezifische Konstruktion politischer Multiplizität in der realistischen Weltanschauung aufgedeckt (Tickner 1992), sondern manchmal sogar - zum Beispiel in der Arbeit von Charlotte Hooper (2001) - direkt die Rolle der gesellschaftlichen Multiplizität bei der Gestaltung von Geschlechterbeziehungen untersucht. Und natürlich dreht sich in der postkolonialen Theorie alles um Andersartigkeit, Hybridität und die Ablehnung unilinearer eurozentrischer Modelle. Sie bildet daher direkt drei der Kernfolgen der Multiplizität ab: Differenz, Kombination und dialektische Veränderung.

Man könnte weitere Beispiele finden, aber der Hauptpunkt sollte klar geworden sein: Möglicherweise ist der fehlende Schlüssel zum verstreuten Puzzle der heutigen IB-Theorie die Bedeutung der Multiplizität für die menschliche Welt. Sobald wir diesen Schlüssel haben, wird deutlich, dass wir bereits alle auf unterschiedliche Weise am gleichen Diskurs teilnehmen. Und wie bereits angemerkt, ist Multiplizität auch die große Idee, die die IB in die Konversation der Geistes- und Sozialwissenschaften einbringt und damit eine Lösung für deren nachhaltige Probleme des methodischen Nationalismus, des Eurozentrismus und des unilinearen Essentialismus bietet.

Und doch zeigen diese beiden Behauptungen auch, dass das Puzzle unvollständig ist. Und damit kommen wir zum zweiten Teil unserer Antwort: der Agenda, die die Multiplizität für die Weiterentwicklung der IB mit sich bringt.

Um die Lücken in den Mittelpunkt zu stellen, erweitern wir die Metapher ein letztes Mal. Wenn das Bild im Puzzle das einer großen Eiche ist, dann muss noch ein Großteil des Außenlaubs (wo die Äste am weitesten reichen und die Bedeutung des Internationalen über die IB hinaus tragen) ausgefüllt werden. Eine bewusst IBverwurzelte Literatur, die die Folgen gesellschaftlicher Multiplizität in die verschiedensten Themenbereiche - z.B. der Soziologie, Ökonomie, Vergleichenden Literaturwissenschaft, Psychologie, Musik, Welternährung - verfolgt, existiert noch 
nicht. ${ }^{2}$ Wenn sich eine solche Literatur herausbildet, werden wir sicher feststellen, dass diese Folgen im Rahmen unterschiedlicher Phänomene variieren. Die Kausalmechanismen, die sie in der Politik produzieren, unterscheiden sich von denen, die sich in der Evolution sozialer Strukturen kristallisieren oder gar in der Literatur oder der Kochkunst. Doch weit davon entfernt, die einheitliche disziplinäre Prämisse (und Verheißung) der IB aufzulösen, soll diese Proliferation sie bereichern und stärken, indem sie die vielfältige Substanz des Internationalen als Dimension der menschlichen Welt freilegt (so wie die interdisziplinären Aktivitäten von AnthropologInnen, GeografInnen und PolitikwissenschaftlerInnen dies für Kultur, Räumlichkeit und Macht tun).

Schließlich beleuchtet diese Perspektive ein weiteres unerwartetes Merkmal unseres Eichenpuzzles: nämlich dass auch noch wichtige Teile von Stamm und Wurzeln fehlen - die die inhaltlichen und theoretischen Definitionen des Internationalen selbst enthalten. Bisher sind nur die politischen (und Teile der wirtschaftlichen) Puzzlestücke des Stammes an Ort und Stelle. Die Wurzeldefinition des Internationalen an sich - in der seine ontologische Voraussetzung identifiziert und theoretisch durchgearbeitet wird - befindet sich noch im Anfangsstadium.

Das Problem wird deutlich, wenn wir an die vier so genannten großen Debatten denken, die in der (angelsächsischen) Mainstream-IB nach wie vor die historische Entwicklung der Disziplin markieren. ${ }^{3}$ Die erste Debatte konzentrierte sich - verständlicherweise angesichts des historischen Kontextes der Zwischenkriegszeit stark auf konkurrierende liberale und realistische Antworten auf die Herausforderung der politischen Multiplizität. Die zweite jedoch, die in den 1960er Jahren stattfand, trat nicht an, um die tiefere Ontologie des Internationalen $\mathrm{zu}$ formulieren. Stattdessen stellte sie einen methodologischen Streit zwischen behavioristischen und traditionellen Ansätzen zu dieser Herausforderung in den Vordergrund. Eine kurze dritte Debatte in den 1970er und 80er Jahren brachte neben den Positionen der Machtpolitik und der Interdependenz, die zuvor das Feld dominiert hatten, einen strukturalistischen Ansatz hervor. Und doch ergab sich aus dieser Entwicklung die grundlegende (Kuhn'sche) erkenntnistheoretische Frage, ob diese Paradigmen vergleichbar waren (Banks 1985). Und schließlich stellte die vierte Debatte ab den 1980er Jahren eine reflektivistische Epistemologie gegen einen rationalistischen Mainstream und öffnete die Tür zu einer Vielzahl von kritischen (poststruktu-

2 Was natürlich existiert, sind zahlreiche lokale Studien in den anderen Disziplinen, die sich - oft brillant - mit der intergesellschaftlichen, globalen oder verstrickten Dimension ihres Fachs auseinandersetzen. Es sind diese Interventionen, die die IB unterstützen könnte, indem sie eine allgemeine theoretische Sprache der Vielfalt und ihrer Folgen zur Verfügung stellt.

3 Diese Geschichte der großen Debatten wird aus verschiedenen Gründen, und zu Recht, kritisiert (Schmidt 1992). Für den hiesigen Zusammenhang ist jedoch nicht ihre historische Richtigkeit entscheidend, sondern die zentrale Rolle, die diese Geschichte dabei spielt, die Reichweite der Disziplin in der Vorstellung von IB-WissenschaftlerInnen zu begrenzen. Und in dieser Beziehung, wie ihre kontinuierliche Reproduktion in anglophonen Textbüchern zeigt, bleibt sie hegemonial. 
ralistischen, feministischen und postkolonialen) Ansätzen, die ihren Ursprung in anderen geistes- und sozialwissenschaftlichen Disziplinen hatten.

Diese Geschichte der großen Debatten zeigt eine Disziplin in einer lebendigen, umstrittenen Evolution. Die Streitigkeiten konzentrieren sich jedoch entweder auf die ursprüngliche Etablierung der IB als Teilgebiet der Politikwissenschaft oder auf den anschließenden Import von Methoden und Erkenntnistheorien aus anderen Disziplinen. In keinem Fall war die Debatte von einem gemeinsamen Versuch getrieben, aus der Tatsache des Internationalen selbst Schlussfolgerungen zu ziehen, seine einzigartigen Eigenschaften $\mathrm{zu}$ beschreiben und seine Folgen für alle anderen Dimensionen der menschlichen Welt zu verfolgen. Der Realismus, das muss man zugeben, ist dem am nächsten gekommen. Aber wie bereits angedeutet, verschmilzt in dessen negativer Definition von Anarchie politische Multiplizität mit der Multiplizität selbst, verdeckt deren tiefer liegende Existenz und blockiert damit auch den Weg zur Aufdeckung ihrer Implikationen jenseits der Disziplin der IB. Es ist daher kein Wunder, dass die IB unter der ständigen Angst um ihre Stellung als Disziplin gelitten haben. Aber es sollte inzwischen auch klar sein, dass dies keine notwendige Zwangslage ist. Multiplizität ist der Schlüssel sowohl für die innere Kohärenz der IB als auch für ihren Beitrag zu den anderen Geistes- und Sozialwissenschaften. Alle menschlichen Praktiken finden im Kontext mehrerer interagierender Gesellschaften statt. Wenn wir diese ontologische Tatsache intellektuell in Besitz nehmen können, dann kann sich der aktuelle Schlüsselpunkt in der Geschichte unseres Feldes noch als Vorbote erweisen, nicht für das „Ende der IB-Theorie“ (Dunne et al. 2013), sondern für ihren lange verzögerten Beginn.

\section{Literatur}

Ashley, Richard 1984: The Poverty of Neorealism, in: International Organization 38: 2, 225-261.

Bacon, Francis 1960: The New Organon and Related Writings (Edited, with an Introduction by, Fulton H. Anderson), New York, NY.

Banks, Michael 1985: The Inter-Paradigm Debate, in: Light, Margot/Groom, A.J.R. (Hrsg.): International Relations: A Handbook of Current Theory, London, 7-26.

Bauman, Zygmunt 1992: Intimations of Postmodernity, London.

Beck, Ulrich 2007: The Cosmopolitan Condition. Why Methodological Nationalism Fails, in: Theory, Culture \& Society 24: 7-8, 286-290.

Bozeman, Adda 1994: Politics and Culture in International History: From the Ancient Near East to the Opening of the Modern Age, 2. Auflage, New Brunswick, NJ.

Brown, Chris 2013: The Poverty of Grand Theory, in: European Journal of International Relations 19: 3, 483-497.

Buzan, Barry/Little, Richard 2001: Why International Relations Has Failed as an Intellectual Project and What to Do About It, in: Millennium 30: 1, 19-39.

Callinicos, Alex 2007: Does Capitalism Need the State System?, in: Cambridge Review of International Affairs 20: 4, 533-49.

Campbell, David 1992: Writing Security. United States Foreign Policy and the Politics of Identity, Manchester. 
Dunne, Tim/Hansen, Lene/Wight, Colin 2013: The End of International Relations Theory?, in: European Journal of International Relations 19: 3, 405-425.

Geertz, Clifford 1993 [1973]: The Interpretation of Cultures. Selected Essays, London. Gregory, Derek/Urry, John (Hrsg.) 1985: Spatial Relations and Social Structures, London.

Guldi, Jo/Armitage, David 2014: The History Manifesto, Cambridge.

Gutas, Dimitri 1998: Greek Thought, Arabic Culture. The Graeco-Arabic Translation Movement in Baghdad and Early Abbasid Society (2nd-4th/8th-10th Centuries), Abingdon.

Halliday, Fred 1994: Rethinking International Relations, Basingstoke.

Hobson, John 2004: The Eastern Origins of Western Civilisation, Cambridge.

Hoffmann, Stanley 1959: International Relations. The Long Road to Theory, in: World Politics 11: 3, 346-377.

Hogg, Richard/Denison, David (Hrsg.) 2006: A History of the English Language, Cambridge.

Hooper, Charlotte 2001: Manly States. Masculinities, International Relations, and Gender Politics, New York, NY.

Kristensen, Peter 2016: Discipline Admonished: On International Fragmentation and the Disciplinary Politics of Stocktaking, in: European Journal of International Relations 22: 2, 243-267.

Kurki, Milja 2017: Spoken Contribution to the Roundtable on "International Relations in the Prison of Political Science", International Studies Association, Baltimore, MD, February $23^{\text {rd }}, 2017$.

Leftwich, Adrian (Hrsg.) 2004: What is Politics?, Cambridge.

Marx, Karl/Engels, Friedrich 1970: The German Ideology. Part One (Edited, with an Introduction, by C.J. Arthur), London.

Matin, Kamran 2013: Recasting Iranian Modernity. International Relations and Social Change, London.

McNeill, John R./McNeill, William H. 2003: The Human Web. A Bird's-Eye View of World History, New York, NY.

Mills, C. Wright 1959: The Sociological Imagination, Oxford.

Megarry, Tim (Hrsg.) 1995: From the Caves to Capital. Readings in Historical and Comparative Sociology, Dartford.

Nisbet, Robert 1969: Social Change and History. Aspects of the Western Theory of Development, London.

Pugel, Thomas 2015: International Economics, 15. Auflage, New York, NY.

Reiter, Dan 2015: Should We Leave behind the Subfield of International Relations?, in: Annual Review of Political Science 18: 1, 481-499.

Rosenberg, Justin 2013: The "Philosophical Premises" of Uneven and Combined Development, in: Review of International Studies 39: 3, 569-597.

Rosenberg, Justin 2016: International Relations in the Prison of Political Science, in: International Relations 30: 2, 127-153.

Rosenberg, Justin/Boyle, Chris 2019: Understanding 2016: Brexit and Trump in the History of Uneven and Combined Development, in: The Journal of Historical Sociology 33: 1, i.E.

Schmidt, Brian 1992: On the History and Historiography of International Relations, in: Carlsnaes, Walter/Risse, Thomas/Simmons, Beth A. (Hrsg.): Handbook of International Relations, London, 3-28.

Schwarz, Roberto 1992: Misplaced Ideas. Essays on Brazilian Culture, London.

Seth, Sanjay 2011: Postcolonial Theory and the Critique of International Relations, in: Millennium 40: 1, 167-183.

Skocpol, Theda 1973: A Critical Review of Barrington Moore's Social Origins of Dictatorship and Democracy, in: Politics \& Society 4: 1, 1-34.

Smelser, Neil 1988: Social Structure, in: Smelser, Neil (Hrsg.): Handbook of Sociology, London, 103-129.

Sylvester, Christine 2007: Whither the International at the End of IR?, in: Millennium 35: 3, 551-573. 


\section{Forum}

Tenbruck, Friedrich 1989: Gesellschaftsgeschichte oder Weltgeschichte?, in: Kölner Zeitschrift für Soziologie und Sozialpsychologie 41: 3, 417-439.

Tickner, Anne 1992: Gender in International Relations. Feminist Perspectives on Achieving Global Security, New York, NY.

Vogel, Ezra 2011: Deng Xiaoping and the Transformation of China, Cambridge, MA.

Walker, Rob 1992: Inside/Outside. International Relations as Political Theory, Cambridge.

Wallerstein, Immanuel 2001: Unthinking Social Science, Philadelphia, PA.

Walzer, Michael 1994: Thick and Thin. Moral Argument at Home and Abroad, Notre Dame, IN.

Wendt, Alexander 1992: Anarchy is What States Make of It. The Social Construction of Power Politics, in: International Organization 46: 2, 391-425.

Wolf, Eric 1982: Europe and the People Without History, Berkeley, CA. 